\title{
Spontaneous Pain, Both Neuropathic and Inflammatory, Is Related to Frequency of Spontaneous Firing in Intact C-Fiber Nociceptors
}

\author{
Laiche Djouhri, Stella Koutsikou, Xin Fang, Simon McMullan, and Sally N. Lawson \\ Department of Physiology, Medical School, University of Bristol, Bristol BS8 1TD, United Kingdom
}

\begin{abstract}
Spontaneous pain, a poorly understood aspect of human neuropathic pain, is indicated in animals by spontaneous foot lifting (SFL). To determine whether SFL is caused by spontaneous firing in nociceptive neurons, we studied the following groups of rats: (1) untreated; (2) spinal nerve axotomy (SNA), L5 SNA 1 week earlier; (3) mSNA (modified SNA), SNA plus loose ligation of the adjacent L4 spinal nerve with inflammation-inducing chromic gut; and (4) CFA (complete Freund's adjuvant), intradermal complete Freund's adjuvant-induced hindlimb inflammation 1 and $4 \mathrm{~d}$ earlier. In all groups, recordings of SFL and of spontaneous activity (SA) in ipsilateral dorsal root ganglion (DRG) neurons (intracellularly) were made. Evoked pain behaviors were measured in nerve injury (SNA/mSNA) groups. Percentages of nociceptive-type C-fiber neurons (C-nociceptors) with SA increased in intact L4 but not axotomized L5 DRGs in SNA and mSNA (to 35\%), and in L4/L5 DRGs 1- $4 \mathrm{~d}$ after CFA (to 38-25\%). SFL occurred in mSNA but not SNA rats. It was not correlated with mechanical allodynia, extent of L4 fiber damage [ATF3 (activation transcription factor 3) immunostaining], or percentage of L4 C-nociceptors with SA. However, L4 C-nociceptors with SA fired faster after mSNA $(1.8 \mathrm{~Hz})$ than SNA $(0.02 \mathrm{~Hz})$; estimated L4 total firing rates were $\sim 5.0$ and $\sim 0.6 \mathrm{kHz}$, respectively. Similarly, after CFA, faster L4 C-nociceptor SA after $1 \mathrm{~d}$ was associated with SFL, whereas slower SA after $4 \mathrm{~d}$ was not. Thus, inflammation causes L4 C-nociceptor SA and SFL. Overall, SFL was related to SA rate in intact C-nociceptors. Both L5 degeneration and chromic gut cause inflammation. Therefore, both SA and SFL/spontaneous pain after nerve injury (mSNA) may result from cumulative neuroinflammation.
\end{abstract}

Key words: dorsal root ganglion; DRG; pain; neuropathic pain; neuroinflammation; C-fiber; spontaneous pain

\section{Introduction}

Chronic neuropathic pain can result from nerve injury/damage and is refractory to analgesic therapy. It is characterized in humans by spontaneous pain, an important, but poorly understood aspect of neuropathic pain, and also by allodynia and hyperalgesia (Bonica, 1990). The clinical symptoms of spontaneous pain include sensations of pins and needles, shooting, burning, stabbing, and paroxysmal (electric shock-like) pain sometimes associated with dysesthesia and/or paresthesia (Attal and Bouhassira, 2004). Spontaneous foot lifting (SFL) behavior is thought to indicate spontaneous pain (Bennett and Xie, 1988; Choi et al., 1994).

Although spontaneous pain seems likely to be caused by spontaneous activity (SA) of neurons in the afferent (perhaps nociceptive) pathway, remarkably no direct evidence links SFL with SA in primary afferents, although a causal link between spontaneous

\footnotetext{
Received Aug. 11, 2005; revised Dec. 5, 2005; accepted Dec. 11, 2005

This work was supported by grants to S.N.L. from the Wellcome Trust and the Biotechnology and Biological Sciences Research Council UK. S.K. was supported by a Bristol University studentship. We thank C. Berry and B. Carruthers for technical assistance and T. J. Biscoe for comments on this manuscript.

Correspondence should be addressed to Dr. Laiche Djouhri, Department of Physiology, University of Bristol, Medical School, University Walk, Bristol BS8 1TD, UK. E-mail: L.Djouhri@bristol.ac.uk.

$X$. Fang's present address: Department of Biological Sciences, University of Warwick, Coventry CV4 7AL, UK. D01:10.1523/JNEUROSCI.3388-05.2006

Copyright $\odot 2006$ Society for Neuroscience $\quad$ 0270-6474/06/261281-12\$15.00/0
}

afferent firing and the development of allodynia and hyperalgesia has been suggested (for review, see Devor and Seltzer, 1999). After peripheral nerve injury, spontaneous firing can occur in both injured A-fibers and intact C-fibers (Boucher et al., 2000; Liu et al., 2000; Wu et al., 2001). However, the questions of whether A- or C-fibers, and whether injured or uninjured fibers, are more important for the generation of spontaneous pain, remain unanswered.

The original spinal nerve (SN) ligation (SNL) model initiated by Kim and Chung (1992) rarely results in reported SFL. We used two SN injury models of neuropathic pain, only one of which gave rise to SFL. These were the following: (1) the SNA (SN axotomy) model in which the L5 SN was ligated and axotomized (this showed little or no ensuing SFL), and (2) the mSNA (modified SNA) model involving SNA plus loose ligation of the L4 SN with chromic gut [this model resulted in SFL (Koutsikou and Lawson, 2002; this study)]. Lee et al. (2003) introduced this L4 loose ligation, although chromic gut ligatures were originally used in the chronic constriction injury (CCI) neuropathic pain model (Bennett and Xie, 1988). The effectiveness of chromic gut ligatures in inducing neuropathic pain-related behaviors has been attributed to induction of an inflammatory response (Maves et al., 1993).

Because SFL is seen only in mSNA rats, it must be associated with damage and/or inflammation to the L4 SN caused by chro- 
mic gut. We therefore hypothesized that this SFL may result from an increase in SA in L4 DRG neurons in mSNA compared with SNA rats. To test this hypothesis, we compared the following between mSNA and SNA rats: (1) the incidence and duration of SFL and (2) the percentage and types of DRG neurons with SA and also the rate of SA measured with intracellular recording in DRG neurons in vivo. This recording method enables unambiguous identification of the types of afferent neuron involved. Chromic gut and complete Freund's adjuvant (CFA) both cause inflammation, albeit in different sites and not necessarily identical. We therefore compare the behavioral and neural responses to intradermal injection of CFA with those found in nerve injury models. Preliminary neuropathic behavioral data were published in abstract form (Koutsikou and Lawson, 2002).

\section{Materials and Methods}

All experimental procedures complied with Home Office United Kingdom Guidelines. Young female Wistar rats $(150-180 \mathrm{~g})$ rats were used throughout. Animals were killed at the end of experiments with an overdose of the anesthetic.

Preparation of neuropathic and inflammatory pain models. SN surgery was performed under sterile and aseptic conditions and deep anesthesia (sodium pentobarbitone; $60 \mathrm{mg} / \mathrm{kg}$, i.p.), and CFA injections were made under halothane anesthesia.

SNA. Seven days before electrophysiological recordings, the SNA was produced by tight ligation with silk (see below) followed by transection of the left L5 SN (Li et al., 2000) [i.e., using a modification of the procedure described originally by Kim and Chung (1992)]. Briefly, the left transverse process of the L6 vertebra was exposed and removed. The ventral ramus of the L5 SN was isolated, tightly ligated with a 6-0 silk suture (Ethicon, Brussels, Belgium; Look, Taunton, UK), and transected (L5 axotomy) just distal to the suture with care taken to avoid damaging the spared L4 SN (Fig. 1). The skin incision was closed with two layers of sutures (subcutaneous and cutaneous), and healing occurred normally in all cases by day 7 after surgery. Abbreviations for axotomized L5 DRGs and the spared ipsilateral L4 DRGs are L5Axot and L4Spar, respectively.

$m S N A$. In addition to L5 axotomy (as above), the ipsilateral L4 SN was loosely ligated (Fig. 1) with a chromic gut suture (5-0; Ethicon; Look). The diameter of the loop around the L4 SN was at least $2 \mathrm{~mm}$ greater than the diameter of the nerve (Lee et al., 2003), which is much looser than that used in the CCI model (Bennett and Xie, 1988). DRGs associated with these axotomized L5 and loose-ligated L4 SNs are abbreviated as L5Axot and L4LL, respectively.

CFA. In an additional group of animals without SNA or mSNA surgery, a unilateral hindlimb inflammation was induced under halothane anesthesia by two $100 \mu$ lintradermal injections of CFA (Sigma, St. Louis, $\mathrm{MO}$ ), one into the plantar surface of the left hindpaw and one beside the left knee. Behavioral tests were performed at 1, 2, 4, and $7 \mathrm{~d}$ after treatment, and electrophysiological recordings were made $1 \mathrm{~d}$ (CFA1 rats) and $4 \mathrm{~d}$ (CFA4 rats) after CFA treatment. The ipsilateral L4/L5 DRGs are abbreviated as L4/5CFA1 ( $1 \mathrm{~d}$ after CFA) and L4/5CFA4 ( $4 \mathrm{~d}$ after CFA). Units with receptive fields on the hip and trunk (i.e., units with receptive fields outside the inflamed areas of the leg) were excluded (Djouhri and Lawson, 2001).

Control animals. These had no previous surgery or CFA treatment. The DRGs are abbreviated as L4/5Norm.

Comparisons were made between nerve-injured SNA and mSNA rats, in which the only difference in treatment was the chromic gut loose ligation on the L4 SN (Fig. 1). We did not use a separate sham-operated group; this approach was supported by previous studies showing that DRG neuronal membrane properties in SNL sham rats were similar to those in unoperated control rats (Ma et al., 2003).

Measurement of damage to L4 SN. Finally, to distinguish between inflammatory effects and physical damage to the L4 SN caused by the chromic gut loose ligation, we examined the effects of different amounts of nerve damage on the different types of behavior. The extent of the L4 damage was estimated by counting the percentage of neuronal nuclei in ipsilateral L4 DRGs that showed immunoreactivity for activation transcription factor 3 (ATF3) $7 \mathrm{~d}$ postoperatively. ATF3 expression is used as a neuronal injury marker, and because its induction was detected only after axotomy and not in naive DRG neurons or after peripheral CFA injection (Tsujino et al., 2000), any neurons that still expressed ATF3 $7 \mathrm{~d}$ after surgery were presumed to be axotomized. To achieve a range of damage to the L4 SN, four SNA rats were operated on by an inexperienced operator under training in this laboratory, who had previous experience of only four of these operations. As expected, these operations resulted in greater inadvertent damage to the L4 SN than with the experienced operator, and enabled us to correlate the different types of behavior in relation to each other and to the extent of L4 fiber damage.

\section{Behavioral tests}

All experimental rats (i.e., SNA-, mSNA-, and CFA-treated rats) maintained good health and showed levels of exploratory activity, feeding activity, and weight gain indistinguishable from those of the unoperated normal rats. Animals with postoperative signs of motor deficits were excluded $(n=3)$. The remaining rats were placed singly in a clear plastic chamber $(22 \times 18 \times 14 \mathrm{~cm})$ on a glass floor at room temperature and, after $10 \mathrm{~min}$ for acclimatization, their postures for standing, walking, and resting were monitored daily up to day 7 postoperation (PO) (see below).

Three behavioral signs thought to represent different components of neuropathic pain [that is, spontaneous pain behavior and two forms of evoked pain behavior (mechanical allodynia and heat hyperalgesia)] were studied as follows. (1) Spontaneous pain: we used SFL as a sign of spontaneous pain after nerve injury and CFA-induced inflammation in 
rat as previously described and justified (Bennett and Xie, 1988; Mao et al., 1992; Choi et al., 1994; Han et al., 2000; Schafers et al., 2003; Lee et al., 2003). For further justification of SFL as a behavioral sign of spontaneous pain in the present study, see Results. (2) Mechanical allodynia (hypersensitivity to a normally innocuous stimulus) was indicated by withdrawal thresholds to innocuous von Frey hairs (Chaplan et al., 1994; Kim et al., 1997). (3) Heat hyperalgesia was indicated by latency of withdrawal from a noxious heat stimulus (Hargreaves analgesymeter) (Bennett and Xie, 1988; Hargreaves et al., 1988; Kim and Chung, 1992).

SFL. Spontaneous foot-lifting duration was measured as the cumulative duration in seconds for which rats lifted their ipsilateral hindfoot accompanied often by aversive behavior (e.g., foot shaking/licking) over $5 \mathrm{~min}$. Foot lifting associated with exploratory behavior, locomotion, body repositioning, and grooming was excluded (Yoon et al., 1996). This was repeated twice (10 min total time) with an interval of $5 \mathrm{~min}$. The contralateral hindfoot was also observed over the same period. For SNA and mSNA rats, SFL duration was monitored (but not recorded) on day 6 PO and recorded on day 7 PO (the day of electrophysiological recording). For CFA-treated rats, SFL duration was monitored and recorded on days 1, 2, 4, and 7 after CFA treatment. The SFL values presented in Figure $2, A$ and $B$, are day 7 values in SNA and mSNA rats, and those presented in Figure 2, $C$ and $D$, are values at 1, 2, 4, and $7 \mathrm{~d}$ after CFA treatment.

von Frey hair withdrawal threshold (mechanical allodynia). Each rat was placed on a metal mesh floor. The plantar surface of each hindpaw was touched for $\sim 2 \mathrm{~s}$ with a series of six von Frey filaments (Touch-Test; North Coast Medical, Morgan Hill, CA) providing forces of 0.6, 1, 2, 4, 6, and $8 \mathrm{~g}$, starting with $2 \mathrm{~g}$. The diameters of these filaments are $0.22,0.25$, $0.3,0.36,0.4$, and $0.42 \mathrm{~mm}$, respectively. The $50 \%$ withdrawal threshold was determined by following the up-down method described by Chaplan et al. (1994). A minute was allowed between tests on alternate hindpaws and 2-3 min between subsequent tests on the same hindpaw. A positive response was recorded if the paw was withdrawn during the application of the von Frey filament, or immediately after its removal. The forces that never produced a positive response (withdrawal) in the contralateral foot and in normal untreated rats were 1.0 and $2.0 \mathrm{~g}$ in all animals and $4.0 \mathrm{~g}$ in some animals. Thus responses to 1.0 and $2.0 \mathrm{~g}$ are considered to be allodynic, because they do not evoke a positive response in normal rats or contralateral hindlimbs. This test was applied to SNA and mSNA rats but not CFA-treated rats. The term allodynia throughout refers to mechanical allodynia.

Withdrawal latency to a noxious heat stimulus (heat hyperalgesia). A plantar (Hargreaves) analgesymeter (Ugo Basile, Comerio, Italy) (Hargreaves et al., 1988) was used to measure the latency of response to a noxious heat stimulus applied to the plantar surface of the hindpaw. Each rat was placed on a 2-mm-thick glass floor under which the laser radiant heat source was positioned. The stimulus onset activated a timer that was automatically stopped when the evoked paw withdrawal was detected by a photocell. Three latency measurements were taken and averaged for each hindpaw during each session of testing. A reduced response latency to a normally noxious heat stimulus is considered to represent hyperalgesia. The hindpaws were tested alternately with at least 5 min intervals between consecutive stimuli on the same hindpaw. This test was performed on SNA and mSNA rats only. The term hyperalgesia throughout refers to heat hyperalgesia.

\section{Electrophysiological recordings}

Rats were deeply anesthetized (sodium pentobarbitone; $60 \mathrm{mg} / \mathrm{kg}$, i.p., followed by $10 \mathrm{mg} / \mathrm{kg}$, i.v., per hour as needed). Full details of the animal preparation were as reported previously (Fang et al., 2002, 2005b). They are given here briefly. The paraffin pool temperature at the DRG was maintained at $30 \pm 2{ }^{\circ} \mathrm{C}$. Intracellular recordings from somata of both the left L5 and L4 DRG neurons (Fig. 1) were obtained using sharp glass microelectrodes filled with either $\mathrm{KCl}(3 \mathrm{M}$, St. Paul, MN) or fluorescent dye in $1 \mathrm{M} \mathrm{KCl}$ or $0.1 \mathrm{M} \mathrm{LiCl}$ (Fang et al., 2002). There were no differences in the firing properties of neurons that were recorded with electrodes filled with dye or those filled with $\mathrm{KCl}$ only. The electrode was driven in $1 \mu \mathrm{m}$ steps through the DRG, and for each membrane potential obtained, the dorsal root was stimulated through bipolar platinum elec- trodes (Fang et al., 2002) (Figure 1). Somatic action potentials (APs) evoked by these stimuli, as well as any spontaneously occurring spikes, were recorded on line with a CED (Cambridge Electronics Design, Cambridge, UK) 1401 plus interface and the Spike 2 programs (Cambridge Electronics Design). For each neuron, the membrane potential, conduction velocity $(\mathrm{CV})$, and the presence and frequency of SA were analyzed off-line. All neurons showing an injury discharge after penetration were discarded from this survey. $\mathrm{CV}$ was measured from the latency to the onset of the evoked somatic AP and the conduction distance (typically $4-14 \mathrm{~mm}$ ) to the position of the neuron in the DRG (Djouhri and Lawson, 2001). All neurons were classified according to their dorsal root CVs as $C(\leq 0.8 \mathrm{~m} / \mathrm{s})$, $A \delta(1.5-6.5 \mathrm{~m} / \mathrm{s})$, or $A \alpha / \beta$ at $>6.5 \mathrm{~m} / \mathrm{s}$ on the basis of previously recorded rat dorsal root compound AP recordings (Fang et al. 2002). Muscle afferents were classified the same way. We assumed that $\mathrm{CV}$ s of groups I plus II muscle afferent are similar to those of $A \alpha / \beta$-fibers.

To improve stability during recording, a silver platform was placed beneath the DRG under study, and all animals were paralyzed with a muscle relaxant, pancuronium $(0.5 \mathrm{mg} / \mathrm{kg}$, i.v. $)$. The muscle relaxant was always accompanied by an additional dose $(10 \mathrm{mg} / \mathrm{kg}$, i.v. $)$ of the anesthetic, and these two substances were administered at these doses at regular intervals (approximately hourly). These anesthetic doses were the same as those that induced deep areflexic anesthesia during the preceding period $(2-3 \mathrm{~h})$ of animal preparation. End-tidal $\mathrm{CO}_{2}$ was kept at $\sim 3.5-4 \%$ by adjusting the rate and stroke volume of the respiratory pump. The blood pressure remained stable in the range of $80-100$ $\mathrm{mmHg}$ throughout, indicative of no reduction in the depth of anesthesia at any stage in these experiments.

Spontaneous activity. By spontaneous firing, we mean ongoing firing that is not in response to a known stimulus. However, it may include firing evoked by unknown (nonmechanical) stimuli, such as inflammatory mediators. SA was assessed before modality testing to avoid the search stimuli influencing such firing. In all neurons, the assessment period for SA was initially $2 \mathrm{~min}$. In SNA-, mSNA-, and CFA-treated rats (CFA1 and CFA4), for any L4/L5 DRG neuron that showed SA during this initial observation period, the recording time was extended up to 5 min to calculate the rate of spontaneous firing. These records enabled us to test the hypothesis that SFL might be related to the proportion of neurons with SA. They also enabled us to determine the firing rate of this SA.

Neurons were classified as showing SA only if their firing was clearly distinct from injury discharge and was not attributable to normal ongoing activity. The following exclusions were made: (1) C-cooling units $(n=4)$ were excluded from analyses of "nociceptive-type" neurons, because many normally fire at room temperature; they were, however, included in the "All cells" control group for comparison with axotomized L5 neurons whose sensory receptive properties could not be examined; (2) any neuron with short lasting $(<30 \mathrm{~s})$ high-frequency "injury discharge" caused by impalement; and (3) normal muscle spindle afferents and axotomized units with muscle spindle-like firing rates (see below).

Muscle spindle afferents in intact (including CFA-treated) rats usually had a high stretch-evoked firing rate that was influenced by muscle manipulation. The ongoing firing resulted from the leg being fixed in an extended position (required for stability of recording when testing receptive fields) and thus causing stretch of flexor muscles; the muscle relaxant enhances the passive stretch of muscle spindles. Some muscle spindle afferents showed no ongoing firing but were activated by muscle manipulation. They did not respond to skin stimulation but did respond to mechanical vibration $(>100 \mathrm{~Hz})$. Assessment of SA in such units with ongoing firing was not possible, and all muscle spindle afferents were therefore excluded. For comparability, axotomized $A \alpha / \beta$ units with ongoing firing rates $>10 \mathrm{~Hz}$, a rate normally seen only in muscle spindle units, were also excluded on the assumption that they innervated muscle spindles before axotomy. This assumption is supported by the following: (1) SA develops in axotomized muscle but not axotomized cutaneous afferent neurons (Michaelis et al., 2000), and (2) we never see intact $\mathrm{A} \alpha / \beta$-fiber neurons (except muscle spindle afferents) that fire at $>10 \mathrm{~Hz}$ under any conditions (including inflammation); (3) rapidly conducting (types I and II equivalent to $A \alpha / \beta$ ) muscle afferents are proprioceptive not nociceptive (for review, see Lawson, 2005). Thus, any axotomized 
$\mathrm{A} \alpha / \beta$-fiber units that fire spontaneously at rates $>10 \mathrm{~Hz}$ are likely to be muscle spindle afferents. Nonetheless, any that fired spontaneously at $<10 \mathrm{~Hz}$ would have been included, and indeed the spontaneous firing rate of most axotomized muscle afferents was $<10 \mathrm{~Hz}$ (Michaelis et al., 2000). Thus, although we excluded intact muscle spindle units, we probably included most of those with SA after axotomy. This may have resulted in a slight overestimate of $A \alpha / \beta$ units with SA after axotomy. However, any change in axotomized neurons would have been the same in SNA and mSNA rats and therefore are unlikely to be major contributors to the behavioral differences between these two groups. Our percentages of $A \alpha / \beta$ neurons with SA are similar to those in other studies (see Results).

Sensory receptive properties. After assessment of SA, each neuron with a dorsal root-evoked somatic spike was tested for sensory modality with natural search stimuli. For full methods of classification of sensory properties, see Fang et al. (2002, 2005b). Briefly, intact neurons were classed as low-threshold mechanoreceptive (LTM) units if they responded to innocuous mechanical stimuli (e.g., light brush, light pressure with a blunt object, and/or vibration). Such light pressure caused a slight deformation of the skin inside the experimenter's wrist and resulted in a sensation of touch. C-cooling units were usually spontaneously active at room temperature, and were activated by a cold stimulus (see below) and inhibited by radiant warming but did not respond to mechanical stimuli. Nociceptive units were identified from their responses to noxious stimuli that were applied only to units that failed to respond to any of the above non-noxious stimuli. Noxious stimuli included noxious mechanical (e.g., needle, fine- or coarse-toothed forceps) and noxious thermal stimuli (noxious heat with hot water at $50-65^{\circ} \mathrm{C}$ and noxious cold, a cooled metal rod or ice). The hot water was applied in a jet from a $5 \mathrm{ml}$ syringe on the skin area under investigation. The ice was applied for $\sim 20-30 \mathrm{~s}$ and the cooled metal rod applied for $\sim 1 \mathrm{~s}$, immediately after being taken out of a $-10^{\circ} \mathrm{C}$ freezer. Applying each of these stimuli except the ethyl chloride spray to the skin inside the experimenter's wrist was painful.

Nociceptive neurons had either C- or A-fibers. They were classed as follows: (1) high-threshold mechanoreceptive (HTM) units with cutaneous receptive fields that responded to noxious mechanical but not noxious thermal stimuli, or with deep (subcutaneous) mechanical receptive fields that were not tested with noxious thermal stimuli, (2) mechanoheat units with cutaneous receptive fields that responded to noxious mechanical stimuli and also promptly to a single application of noxious heat (see above), and (3) mechano-cold units that responded to both noxious mechanical and noxious cold stimuli. C- and A $\delta$-fiber nociceptive neurons included all the above neuronal types, but all $\mathrm{A} \beta$ nociceptive neurons included in this study were HTMs.

Unresponsive units were those C- or A $\delta$-fiber units for which no receptive field was found despite an extensive search with non-noxious and noxious mechanical and thermal stimuli. They were probably nociceptive units with inaccessible receptive fields or very high-threshold nociceptors (Fang et al., 2005b). Unlike these fully tested unresponsive units, units lost before full sensory testing was complete were called "unidentified"; some of these had been fully tested with non-noxious and noxious mechanical and were mechano-insensitive, but because they were not tested with thermal stimuli we could not exclude the possibility that they might have been specific cold or heat nociceptors. Unresponsive and unidentified neurons with $\mathrm{C}$ - and $\mathrm{A} \delta$-fibers in this study all had long AP durations typical of nociceptors (Djouhri et al., 1998; Fang et al., 2005b) and were therefore considered likely to be nociceptive and are classed in this paper as "nociceptor-type." This classification is supported by the following: (1) their slow AP kinetics and the slower kinetics of nociceptors relative to LTMs in these DRGs (Fang et al., 2005b) and (2) the high levels of trkA in $\mathrm{C}$ and $\mathrm{A} \delta$ unresponsive neurons indicating their similarity to nociceptors (Fang et al., 2005a). Because AP durations in C- and $\mathrm{A} \delta$-units are not increased 1 week after axotomy (L. Djouhri, X. Fang, S. McMullan, and S. N. Lawson, unpublished observations), it is unlikely that damaged units were misclassified as nociceptors on this basis. In contrast, some unidentified $\mathrm{A} \alpha / \beta$ units had fast kinetics, suggesting that they were LTM units, because most (80\%) $\mathrm{A} \alpha / \beta$ neurons in rat lumbar DRG are LTMs (Djouhri and Lawson, 2004). In this study, therefore, unresponsive or unidentified $\mathrm{A} \alpha / \beta$-fiber units were excluded, and noci- ceptive, unresponsive and unidentified neurons with C- and A $\delta$-fibers were classified under the umbrella term "nociceptive-type" neurons and referred to throughout as nociceptors.

If fibers are injured and do not conduct, it is clearly not possible to determine their nociceptive or LTM receptive properties. For intact nociceptive and LTM neurons in SNA, mSNA, and CFA rats, there was no noticeable difference between the control and experimental DRGs either in the protocols of natural stimuli required to activate them, or in their adequate stimuli. Thus, intact nociceptors were clearly different from intact LTMs in all models studied. It therefore seems unlikely that thresholds of nociceptive neurons could have dropped enough to become incorrectly classified as LTMs, although we did not record mechanical thresholds quantitatively. In the C-fiber range, for example, substantial lowering of threshold after treatment should result in a higher percentage of units being classed as C LTMs than normal ( $\sim 9 \%)$, but this was not seen.

Neither behavioral tests nor electrophysiological recordings were performed blindly for the following reasons. Deformation of the affected foot in the mSNA group and increased girth and redness of the affected foot (inflamed) in the CFA group made these groups easily recognizable in behavioral tests, making blind studies impossible. Sometimes the chromic gut was visible to the experimenter during exposure of the DRG for electrophysiological recording. This is because we expose both L4 and L5 DRGs before recording. Exposure of the L5 DRG, especially removal of bone beneath the DRG to enable placement of the silver platform (to improve stability during recording), may sometimes expose the ends of the chromic gut ligation. A CFA-treated hindfoot was also easily recognizable in the electrophysiological studies. Nonetheless, collection of data in electrophysiological experiments was not affected by knowledge of the previous history of the animal, because in all animal groups (1) sampling by the microelectrode was random, because it was driven through the DRG without regard for possible location of particular cell types, and (2) all C-fiber units and all A-fiber units (except those detailed above under SA) that had stable recordings for $\sim 2$ min were assessed for the presence of SA regardless of their properties; (3) the pattern of difference in spontaneous firing rates between the SNA and mSNA groups (see Results) emerged after off-line analysis after the experiments; (4) furthermore, where behavioral or electrophysiological studies were performed by more than one experimenter, similar results were obtained by all experimenters.

Immunostaining for ATF3. On day $7 \mathrm{PO}$, some of the rats used for behavioral studies were given an overdose of anesthetic and fixed by transcardiac perfusion with Zamboni's fixative preceded by normal saline. The ipsilateral and contralateral L4 and L5 DRGs were removed, postfixed for $1 \mathrm{~h}$, and stored overnight in $30 \%$ sucrose buffer at $4^{\circ} \mathrm{C}$. Series of transverse $7 \mu \mathrm{m}$ sections at regular $(280 \mu \mathrm{m})$ intervals throughout each DRG were cut on a cryostat. After blocking endogenous peroxidase activity with an Avidin/Biotin Blocking kit (Vector Laboratories, Burlingame, CA) and preincubating in 10\% normal goat serum (Jackson ImmunoResearch, West Grove, PA) for $30 \mathrm{~min}, \mathrm{ABC}$ immunocytochemistry was performed as previously described in detail (Fang et al., 2005a). Sections were incubated at $4^{\circ} \mathrm{C}$ overnight with a primary polyclonal antibody (at 1:1000) against ATF3 (Santa Cruz Biotechnology, Santa Cruz, CA), which has been fully characterized (Mashima et al., 2001). The secondary antibody (a biotinylated anti-rabbit IgG; 1:200; Vector Laboratories) was applied for $30 \mathrm{~min}$ at room temperature. Negative controls were prepared by excluding the primary antibody.

All ATF3-labeled and unlabeled nuclei in neuronal profiles in three sections taken at regular intervals through the DRG were counted blindly. The overall percentage of neurons positive for ATF3 was calculated for each ganglion.

Bias, selection criteria, and statistics. C-fiber neurons are inevitably underrepresented because of the difficulty of making stable recordings in these small neurons compared with the larger A-fiber neurons as previously discussed (Djouhri et al., 1998). The need to record SA for 2-5 min before determining sensory receptive properties (taking up to $5 \mathrm{~min}$ ) considerably increases the chances of losing a C-fiber neuron before records are complete. However, comparisons within the C-fiber group (such as percentage with SA) are unbiased. Percentages of different types 
of neuron with SA in Figure 5 were compared with two control groups. First, all axotomized (L5) neurons in mSNA rats (including all sampled neurons except $A \alpha / \beta$ muscle spindle afferent-like neurons) were compared with "All cells" from normal rats (including all nociceptors and LTMs except muscle spindle afferents). Second, identified C, A $\delta$, and A $\beta$ nociceptors in (intact) L4 DRGs in SNA and in mSNA rats and in L4/L5 DRGs in CFA-treated rats were compared with similarly identified neurons in normal (L4/L5) DRGs.

Statistical analysis. Because of small $n$ values in some groups, nonparametric statistics were used for comparisons of data from different groups. Contingency table comparisons $(2 \times 2)$ were made with Fisher's exact test in Figures $2 A$ and 5 (All cells comparisons); $\chi^{2}$ test for trend was used for comparison of data in Figure 2C. Multiple pairwise comparisons of percentages of neurons with and without SA between controls (Norm) and the four experimental groups in the Nociceptive groups in Figure 5 were made with Fisher's exact test, with Bonferroni's correction applied to the $p$ value obtained ( $p$ was multiplied by 4 , the number of tests). Comparisons of SFL (see Fig. $2 B$ ) and median SA firing rates (see Fig. $6 A, B)$ were with the Mann-Whitney $U$ test. Linear regression analyses were performed on data in Figures 3, 4, and 7, regression lines included where significant. All tests were made with GraphPad Prism software, version 4 (GraphPad, San Diego, CA), and were two-tailed, unless otherwise stated. Values of $p<0.05$ were considered significant.

\section{Results}

\section{Behavioral signs of spontaneous pain}

None of the animals showed SFL of the contralateral foot. SFL occurred $1 \mathrm{~d}$ PO in both SNA and mSNA rats.

In SNA rats, SFL was much reduced by day 2, whereas in mSNA rats, it continued to increase and was much greater at day 2 than in SNA rats and was sustained at this high level until day 7 PO (S. Koutsikou and S. N. Lawson, unpublished observations). At $7 \mathrm{~d}$ PO, a greater percentage of mSNA than of SNA rats displayed SFL for $>1 \mathrm{~s} / 10 \mathrm{~min}$ [ $80 \%$ (20 of 25 ) compared with $12 \%$ ( 2 of 17$) ; p<0.001$, Fisher's exact test) (Fig. $2 A$ ). Figure $2 B$ shows a significantly $(p<0.001)$ greater median SFL duration in mSNA compared with SNA rats. In a separate series of experiments (S. Koutsikou and S. N. Lawson, unpublished observations), SFL was not seen in rats $(n=6)$ that had L4 chromic gut loose ligation only (i.e., no L5 axotomy). Thus, it seems that in this model the combination of axotomy of one SN (L5) and chromic gut loose ligation of the adjacent SN (L4) in mSNA rats is critical for SFL development.

All CFA-treated rats $(n=11)(100 \%)$ showed SFL on day 1 , whereas only one of six rats showed SFL on day 2 and none on days 4 and 7 after treatment (highly significant; $\chi^{2}$ for trend) (Fig. $2 C)$. Furthermore, the duration of SFL was significantly greater at $1 \mathrm{~d}$ than at 2, 4, and $7 \mathrm{~d}$ after CFA (Fig. 2D). Note that only SFL durations in the five rats in which electrophysiological recordings were made are included in Figure 2, to compare this with spontaneous neural activity in the same group of rats (see below). Inclusion of the data from the other six rats would increase the significance levels seen in Figure $2 C$.

\section{Relationships between allodynia, hyperalgesia, and SFL after nerve injury}

To ensure that SFL after nerve injury is not an evoked response attributable, for example, to allodynia, linear regression analysis was performed between three different types of behavior (Fig. 3; for details of analysis, see legend). In SNA rats, although the maximum SFL was only $1.5 \mathrm{~s}$, there was a correlation between SFL and hyperalgesia (Fig. $3 B$ ) and a similar but not significant $(p=0.056)$ relationship between SFL and allodynia (Fig. $3 A)$. In mSNA rats (SFL range from 4.5 to $22 \mathrm{~s}$ ), there was no correlation of SFL with either allodynia or hyperalgesia (Fig. $3 A, B$ ). In con-

\section{Spontaneous Foot Lifting: Nerve injury at 7days}
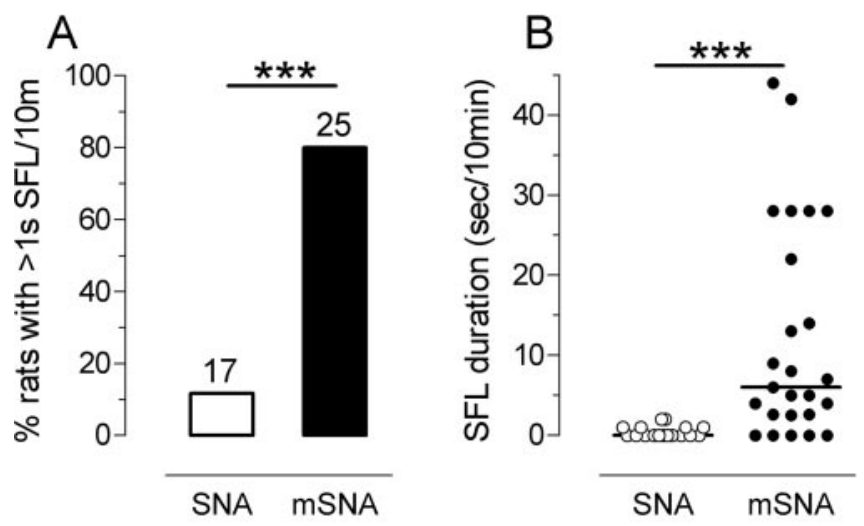

\section{Spontaneous Foot Lifting: CFA at 1-7 days}
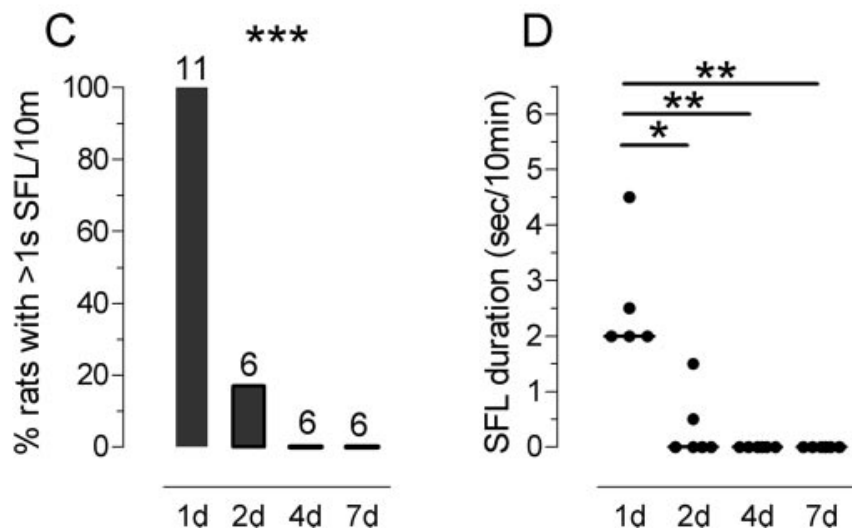

Figure 2. SFL in nerve injury and CFA-treated rats. Numbers above histogram columns in $\boldsymbol{A}$ and $\boldsymbol{C}$ indicate number of rats. $\boldsymbol{A}$ shows that the percentage of $\mathrm{mSNA}$ rats with SFL of $>1 \mathrm{~s} / 10$ min is significantly greater than that of SNA rats ( $p<0.001$, Fisher's exact test). $\boldsymbol{B}$ shows significantly longer SFL duration in mSNA $(n=25)$ than SNA $(n=17)$ rats (Mann-Whitney test). C shows that all rats showed SFL $1 \mathrm{~d}$ after CFA, declining to no rats by $4 \mathrm{~d}$ ( $\chi^{2}$ test for trend). $\boldsymbol{D}$ shows that the amount of SFL is greater at $1 \mathrm{~d}$, declining to none by $4 \mathrm{~d}$ after (FA (Kruskal-Wallis test with Dunn's post test between all groups). ${ }^{*} p<0.05 ;{ }^{* *} p<0.01$; ${ }^{* * *} p<0.001$.

trast, there was a positive correlation between the extents of allodynic and hyperalgesic behaviors (Fig. $3 C$ ) with almost identical regression lines regardless of whether or not the mSNA values were included, suggesting some similarity in mechanisms contributing to these two behaviors. The presence of substantial SFL only in the mSNA rats (Fig. $2 A, B$ ), and the lack of correlation between SFL and the evoked behaviors (allodynia and hyperalgesia) in these rats, suggests different mechanisms for SFL and these two evoked behaviors.

\section{Effects of the extent of L4 SN injury}

The extent of L4 SN injury was determined with ATF3 immunostaining (see lack of positive nuclei in Fig. $4 A$ and two clearly positive nuclei in Fig. $4 B$ ). The relationship of pain behaviors to this injury after L5 axotomy was examined by plotting measures of the different behaviors against the percentages of L4 DRG neuronal nuclei expressing ATF3. In all of the mSNA rats, $>35 \%$ of L4 DRG neurons were ATF3 positive. These rats consistently showed SFL, the duration of which was variable and not correlated with the extent of L4 injury (Fig. $4 C$, filled circles). In SNA 
rats, there was little or no SFL even in those rats with levels of injury in L4 DRGs that were similar to those in mSNA rats $(>35 \%)$ and again no correlation between SFL duration and L4 ATF3 percentage (Fig. $4 C$, open circles). Thus, in the presence of L5 axotomy, it is the L4 chromic gut loose ligation, not merely the extent of L4 fiber damage, that is required for generation of SFL.

In contrast, the extents of evoked behaviors (allodynia and hyperalgesia) were both positively correlated with the percentage of L4 DRG neurons with ATF3 staining (Fig. $4 D, E$ ), indicating an influence on both behaviors of the number of damaged L4 fibers. Linear regression lines between the extent of behavior and ATF3 percentages (Fig. 4D,E, continuous line) for SNA rats were almost perfectly superimposed on those for SNA plus mSNA rats for both allodynia and hyperalgesia (Fig. $4 B, C$, dotted line) (that is, adding the mSNA data did not alter significantly either the slope or elevation of these lines). Thus, the few SNA rats with more extreme inadvertent mechanical damage $(>35 \%$ ATF3) had evoked behaviors that were as great as those in mSNA rats with similar amounts of L4 damage caused by the chromic gut. This is in clear contrast to SFL. We interpret this to mean that, after L5 axotomy, the degree of L4 injury is a major determinant of the extents of allodynia and hyperalgesia, regardless of whether L4 injury is caused directly by loose ligation of the L4 SN, or inadvertently as a consequence of L5 axotomy alone. If this is correct, then the greater extents of these two types of evoked behavior in mSNA compared with SNA rats would have resulted primarily from the consistently greater mechanical damage to $\mathrm{L} 4 \mathrm{SN}$ caused by the chromic gut ligation. Thus, it appears that SFL depended on the presence of chromic gut, most likely the inflammatory effects (see Discussion), whereas the evoked behaviors depended heavily on the extent of L4 SN damage.

\section{Electrophysiological records of SA}

Recordings were made in 191 identified LTMs. In control DRGs, none of 2 A $\delta$ LTMs or of 31 A $\alpha / \beta$ LTMs showed SA. After nerve injury, in L4 DRGs, no A $\delta$ LTMs were recorded in either SNA or mSNA models. There was a slight, but not significant, increase in the percentage of $A \alpha / \beta$ LTM neurons showing SA (13\% of 30 units in SNA, and $10 \%$ of 30 units in mSNA). The firing rates of these $A \alpha / \beta$ LTMs were low and similar in SNA (range, 0.01-0.03 $\mathrm{Hz}$ ) and in mSNA (range, $0.01-0.02 \mathrm{~Hz}$ ) rats. One day after CFA, only 1 A $\delta$ LTM was recorded, and this showed SA with a firing rate of $0.4 \mathrm{~Hz}$, and none of the $2 \mathrm{~A} \alpha / \beta$ LTMs showed SA. Four days after CFA, none of the 8 A $\delta$ LTMs and only 1 of the $87 \mathrm{~A} \alpha / \beta$ LTMs showed SA (rate, $0.01 \mathrm{~Hz}$ ). Because the SA was similar in

\section{B SFL vs hyperalgesia}
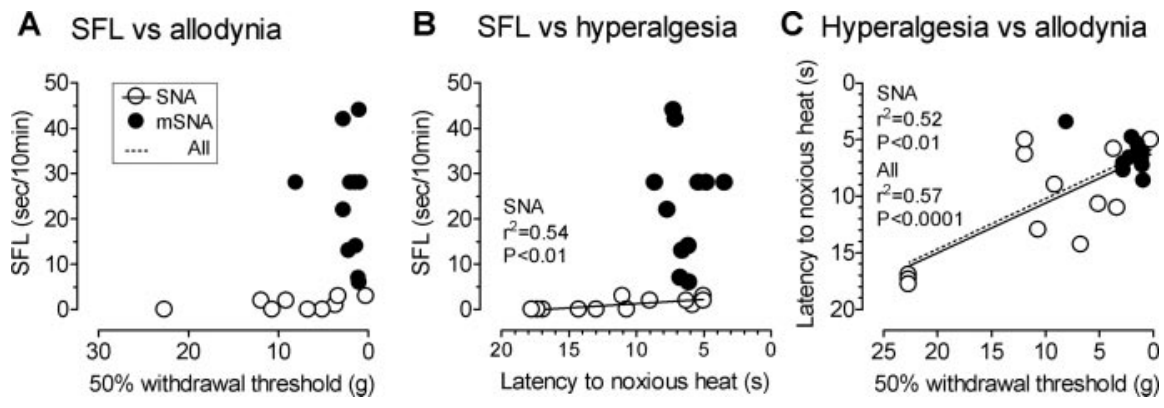

Figure 3. Correlation between behavioral tests. Relationships between extents of different types of behavior: SFL versus heat hyperalgesia $(\boldsymbol{A})$, SFL versus mechanical allodynia $(\boldsymbol{B})$, and heat hyperalgesia versus allodynia ( $\boldsymbol{C}$. The $50 \%$ withdrawal threshold $(\boldsymbol{B}$ and $\boldsymbol{C}$ ) was determined using von Frey filaments as a measure of mechanical allodynia. The direction of the axes shows more circles are data from mSNA rats. Linear regression analysis was performed on SNA and mSNA separately in all cases, and on both ether for allodynia versus hyperalgesia (C); regression lines (solid for SNA and dotted for SNA plus mSNA) are shown only for was not correlated with the extent of SFL in mSNA rats $(\boldsymbol{B})$. The extents of allodynic and hyperalgesic behaviors were correlated, however, for SNA rats alone and for all rats $(\boldsymbol{C})$.

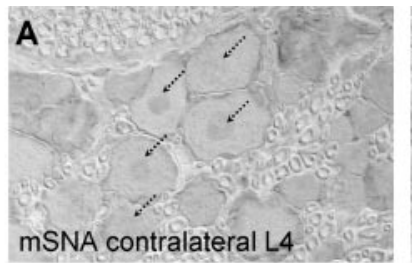

C SFL vs L4 ATF3

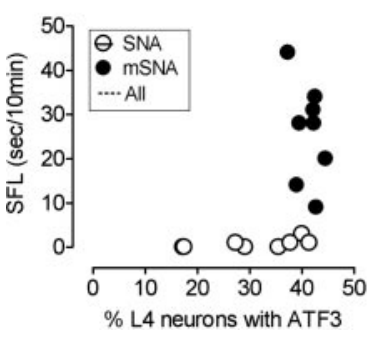

D Allodynia vs L4 ATF3

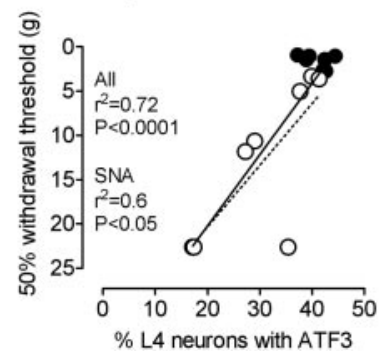

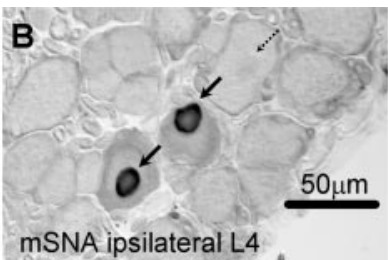

E Hyperalgesia vs allodynia

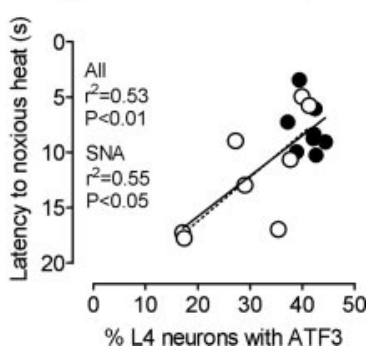

Figure 4. Pain-related behaviors versus extent of $L 4$ SN injury (ATF3). $\boldsymbol{A}, \boldsymbol{B}$, Interference contrast images of examples of ATF3 staining in mSNA rats in L4 DRGs. $\boldsymbol{A}$, Contralateral with no positive nuclei. $\boldsymbol{B}$, Ipsilateral with two positive nuclei; overall, $>40 \%$ per $\angle 4$ DRG neurons expressing ATF3) and SFL (C), mechanical allodynia (D), or heat hyperalgesia (E). Linear regression analysis was performed on SNA and mSNA data separately in all cases, and on both together in graphs $\boldsymbol{B}$ and $\boldsymbol{C}$. Allodynia and hyeralgesia but not SFL were positively correlated with the extent of mechanical damage to the L4SN measured as the percentage $L 4$ neuronal nuclei with ATF3. The dotted arrows $(\boldsymbol{A}, \boldsymbol{B})$ indicate neurons, the neurons of which were unlabeled with ATF3, whereas the solid arrows $(\boldsymbol{B})$ indicate two nuclei labeled for ATF3.

magnitude in A $\alpha / \beta$ LTMs in SNA and mSNA, it was unlikely to contribute greatly to SFL seen only in mSNA rats. Therefore, for the subsequent (main) analyses of intact neurons, we focus exclusively on nociceptors.

Because the only difference between the SNA and mSNA models was the L4 loose ligation, the SFL in mSNA must result from changes in firing properties of L4 neurons in mSNA compared with SNA rats. We therefore examined electrophysiologically the SA in L4 neurons in these two models, and to enable our data to be compared with those from other studies (see below), we also examined SA in axotomized L5 neurons of mSNA rats (Fig. 5). Finally, to enable comparison with a model of inflammatory pain, we recorded SA in L4/L5 DRG neurons in CFA1 and CFA4 rats. In addition to the above LTMs, recordings were made in a total of 719 DRG neurons classified by their dorsal root CVs 

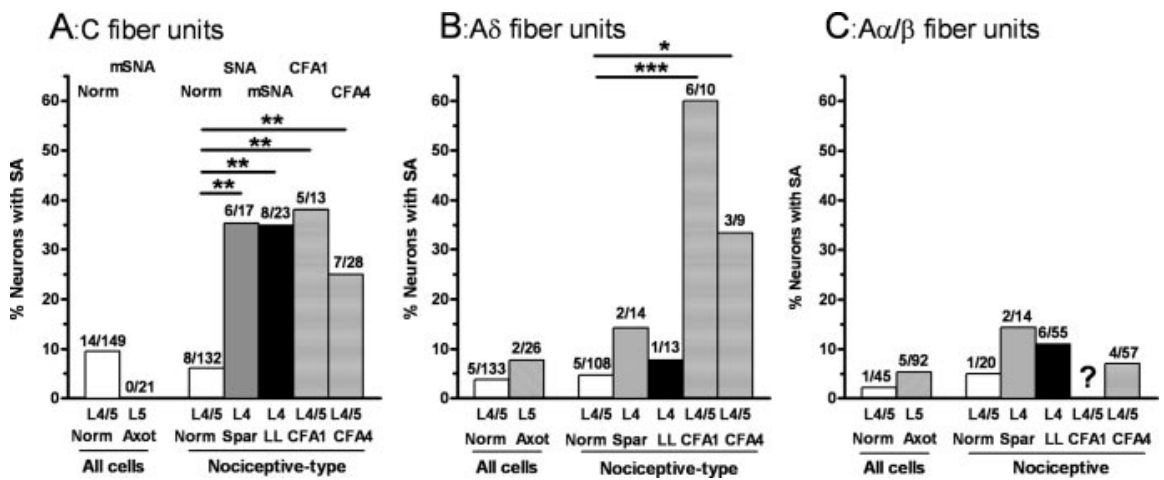

Figure 5. Percentages of $\mathrm{C}$ - and $\mathrm{A}$-fiber neurons with $\mathrm{SA}$ in different pain models. $\boldsymbol{A}, \boldsymbol{C}$-fiber neurons. $\boldsymbol{B}, A \delta$-fiber neurons. $\boldsymbol{C}$, $A \alpha / \beta$-fiber neurons. The model above the relevant column in $A$ relates to all graphs. SNA means L5 SN axotomy 1 week previously; mSNA means SNA plus L4 loose ligation of the L4 SN 1 week previously; CFA1 and CFA4 mean CFA-induced hindlimb inflammation 1 and 4 d previously. Below each histogram is indicated the DRG from which recordings were made. L4/5Norm, L4 and L5 DRGs in untreated rats; L5axot, L5 DRGs after L5 SN axotomy in mSNA rats; L4Spar, ipsilateral L4 in SNA rats; L4LL, ipsilateral L4 in mSNA (with loose ligation); L4/5 CFA1 and L4/5 CFA4, L4 and L5 DRGs in CFA rats 1 and $4 \mathrm{~d}$, respectively, after CFA injection. The left two columns show all neurons from control L4/5 DRGs (L4/5Norm) and axotomized L5 DRGs (L5Axot) in $\boldsymbol{A}$ and $\boldsymbol{B}$ but exclude muscle spindle afferents (first column, L4/5Norm) and muscle spindle afferent-like units (second column, L5Axot) in $C$. The five columns in the right-hand block relate to intact neurons; here, only nociceptors/nociceptive-type neurons are shown, because LTMs did not show increased SA (see Results). These columns show neurons from control (L4/5Norm), SNA- (L4Spar), mSNA- (L4LL), and CFA-treated (L4/5CFA1 and CFA4) rats. In C, the question mark indicates that the proportion of A $\alpha / \beta$ nociceptors with SA was not recorded. The horizontal lines above the graphs link columns between which there was a significant difference Fisher's exact test for $2 \times 2$ contingency tables, followed by Bonferroni's correction. Levels of significance are indicated as follows: ${ }^{*} p<0.05 ;{ }^{* *} p<0.01$; ${ }^{* * *} p<0.001$.

and, where possible, sensory receptive properties. The breakdown of the numbers in each group is shown in Figure 5. A substantial number of C-fiber neurons was recorded in the treatment groups $(n=102)$ and control groups $(n=149)$ despite the difficulty of making intracellular recordings in such neurons. This 102 is made up of $n=21$ in L5 in mSNA, $n=17$ in L4 in SNA, $n=23$ in L 4 mSNA, $n=13$ in CFA1, and $n=28$ in CFA4. Because only a proportion of these neurons in any treatment group (for example, about one-third in SNA and mSNA rats) fired spontaneously, in any one treatment group, the numbers of C-fiber neurons recorded with SA was $<10$ (between 5 and 8). The numbers of rats used for electrophysiology were as follows: 14 mSNA, 7 SNA, 5 CFA (1 d), 26 CFA (4 d), plus 106 control, untreated rats. Many of the rats including most of the control rats provided data for other studies as well.

\section{Percentages of neurons with spontaneous activity}

The percentages of $\mathrm{C}-, \mathrm{A} \delta$-, and $\mathrm{A} \alpha / \beta$-fiber neurons with SA in DRGs of different treatment groups are shown in Figure 5. Because the L5 SN is treated the same (tight ligation with silk followed by axotomy) in both SNA and mSNA rats, and because chromic gut ligature on the L4 SN in mSNA rats is several millimeters from the central stump of the L5 SN, it is unlikely that firing properties of axotomized L5 DRG neurons differ between the two models. The following data show clear differences in the L4 DRGs that could account for the SFL in mSNA but not SNA rats.

\section{Axotomized neurons in L5 DRGs}

Axotomized L5 C-fiber neurons showed no SA (Fig. 5A) consistent with previous studies (Liu et al., 2000). Any changes in SA in L5 neurons are unlikely to contribute to the much greater SFL in mSNA rats; because the L5 SN was axotomized in both models, the SA is likely to be similar in the two models. Compared with untreated controls, axotomy of L5 SN (mSNA rats) resulted in no significant change in percentage of axotomized $A \delta$-fiber or $A \alpha / \beta$-fiber neurons with SA (Fig. $5 B, C$, first two columns); overall, there was an increase to $6 \%$ (not significant) for all A-fiber neurons (data not shown). This is similar to the $8 \%$ value reported previously in an SNA model (Ma et al., 2003) and suggests that a similar percentage of axotomized L5 A-fiber neurons have SA in SNA and mSNA rats. Note that muscle spindle afferents were excluded from this control group, and axotomized $\mathrm{A} \alpha / \beta$-fiber units firing at $>10 \mathrm{~Hz}$ were also excluded. We do not exclude a role in neuropathic pain for fast conducting muscle afferent units that develop SA after nerve injury (Michaelis et al., 2000). However, because the L5 SN was axotomized in both SNA and mSNA rats, any SA in L5 neurons cannot account for the main behavioral difference between the two groups, namely, the SFL.

\section{Neurons in ipsilateral L4 DRGs}

Figure $5 A$ shows that, compared with control rats, there was a similar large and significant increase in the percentage of nociceptive L4 C-fiber neurons with SA in both SNA (gray column) and mSNA (black column) rats (from 7 to $35 \%$ in both cases; Fisher's test, $p<0.01$ ). Thus, the percentage/ number of these C-fiber neurons with SA could not, per se, account for the clear SFL seen in mSNA rats. Of the $17 \mathrm{C}$-fiber neurons in SNA rats, 9 were nociceptive, 5 were unresponsive, and 3 were unidentified (not fully tested) units. Of the $23 \mathrm{C}$-fiber neurons in mSNA rats, 15 were nociceptive, 3 were unresponsive, and 5 were unidentified units. For A $\delta$-fiber neurons, 10 of the 14 units in SNA rats were nociceptive, 2 were unresponsive, and 2 were unidentified, whereas 9 of 13 units in mSNA rats were nociceptive and the remaining 4 were unidentified units.

Of the eight C-fiber nociceptive-type neurons with SA in mSNA rats (Fig. 5A, black column, L4LL), five were nociceptive and three were unidentified (not fully tested). The latter three neurons had electrophysiological properties (action potential variables) that were very similar to those previously reported by Fang et al. (2005b) for nociceptive or unresponsive neurons and different from those of $\mathrm{C}$ low-threshold mechanoreceptors. They are unlikely to have been axotomized, because no L5 C-fiber neurons showed SA after axotomy (Fig. $5 A$, column 2). They were therefore probably nociceptive units with inaccessible receptive fields or very high-threshold nociceptors (see Materials and Methods). These eight $\mathrm{C}$-fiber neurons with SA were recorded in five of eight mSNA rats from which recordings were made. Of the six units with SA in SNA rats (Fig. 5A, gray column, L4Spar), four were nociceptive and two were unresponsive. These units were recorded from all six SNA rats in which recordings were made.

The absence of SA in axotomized L5 C-fibers (Fig. 5A) (all recorded in mSNA rats) suggests that even the unidentified L4 C-fiber units with SA in SNA and mSNA rats are likely to have intact fibers.

\section{L4/L5 neurons after CFA inflammation}

A potential cause of the C-fiber SA in L4 DRGs was inflammation attributable to Wallerian degeneration of axotomized L5 periph- 
eral fibers within the same peripheral nerve (sciatic) (see Discussion). We therefore examined whether inflammation alone, albeit applied to the periphery and not the nerve trunk, could have a similar effect. Because we found SFL $1 \mathrm{~d}$ but not $4 \mathrm{~d}$ after CFA, we examined whether C-fiber SA was greater on day 1 than day 4 .

Both 1 and $4 \mathrm{~d}$ after CFA treatment, the percentage of C-fiber nociceptors with SA was significantly increased compared with untreated control rats from $7 \%$ (control) to $38 \%$ ( $1 \mathrm{~d}$ ) and $25 \%$ (4 d) (Fig. 5A). These values were of a similar magnitude to those in $\mathrm{L} 4 \mathrm{C}$ nociceptors in nerve-injured rats $7 \mathrm{~d} \mathrm{PO}$ (Fig. 5A, L4Spar and L4LL). This is consistent with inflammatory processes being involved in generation of SA in C-fiber neurons after both nerve injury and inflammation.

However, after CFA treatment, unlike $7 \mathrm{~d}$ after nerve injury, there was also a significant increase in percentage of $A \delta$-fiber nociceptors with SA (from $6 \%$ in untreated to $60 \%$ at $1 \mathrm{~d}$ and to $33 \%$ at 4 d) (Fig. $5 B$ ). A $\beta$-fiber nociceptors were not evaluated in CFA1 animals, but there was no increase in CFA4 rats. The percentage of A $\delta$ nociceptors with SA was thus greater 1 and $4 \mathrm{~d}$ after CFA than $7 \mathrm{~d}$ after nerve injury. One explanation could be similar accessibility of nociceptive terminals of both $\mathrm{A} \delta$ - and C-fibers to inflammatory mediators in the periphery, but lower accessibility of myelinated than unmyelinated fibers to such mediators within the nerve.

\section{Membrane potentials of C-fiber neurons with SA}

Fluctuations in membrane potential in somata of axotomized A-fiber but not C-fiber neurons have been suggested as a cause of SA (Amir et al., 1999). We did not see such fluctuations in L4 C-fiber neurons, suggesting that the SA in these neurons may originate peripheral to the soma. Furthermore, there were no significant differences in median membrane potentials of C-fiber neurons between (1) units with SA $(-50 \mathrm{mV})$ and without SA $(-48 \mathrm{mV})$ in mSNA rats $(p>0.05$; Mann-Whitney $U$ test $)$ or between (2) units recorded in L4/L5 DRGs from untreated rats $(-46 \mathrm{mV}), \mathrm{L} 5 \mathrm{DRG}$ in SNA rats $(-50 \mathrm{mV}), \mathrm{L} 4 \mathrm{DRG}$ in either mSNA $(-50 \mathrm{mV})$ and SNA rats $(-51 \mathrm{mV})$ (Kruskal-Wallis test followed by Dunn's post test). These are all similar to the median value $(-50 \mathrm{mV})$ reported previously for normal rats (Fang et al., 2005b).

\section{Rate of SA in L4 nociceptive-type neurons in SNA and mSNA rats}

\section{C-fiber neurons}

The data presented above indicate that, unexpectedly, the percentage of L4 C-fiber neurons with SA was similar in SNA and mSNA rats and therefore could not alone account for the SFL in mSNA rats. The question "Why do mSNA and SNA rats show different SFL behavior?" therefore remained unanswered. This led us to formulate the hypothesis that the rate of firing may be greater in mSNA than SNA rats. Analysis of the recordings of SA that were already available indicated this to be the case, and additional experiments confirmed this finding. Accordingly, we present these rates below.

The median SA rate in these neurons in mSNA rats was significantly $(p<0.01)$ higher (median, $1.8 \mathrm{~Hz}$; range, $0.3-3.8 \mathrm{~Hz}$ ) than in SNA rats $(0.017 \mathrm{~Hz}$; range, $0.01-1.3 \mathrm{~Hz})$, a $>100$-fold increase (Fig. 6A, Table 1). Medians are the appropriate measure for statistical comparison given the distribution of values and numbers of C-fiber units analyzed. Nonetheless, the total input to the spinal cord could only be estimated by adding the firing rates of all units with SA (see below). To make this estimate, the mean C-fiber firing rates are needed; accordingly, these are given

\section{Spontaneous firing in nociceptors with SA}

A: C-fiber neurons

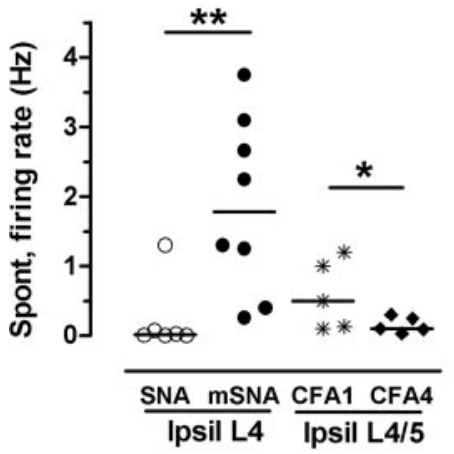

C: SNA L4 C HTM $0.024 \mathrm{~Hz}$
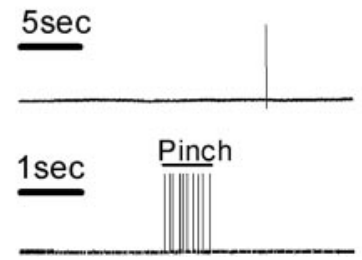

B: A-fiber neurons

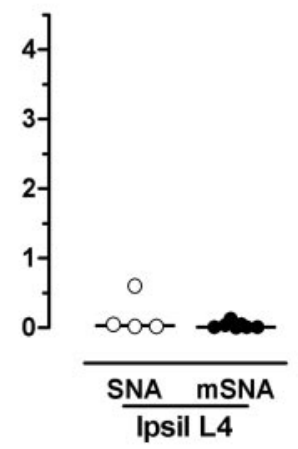

D: mSNA L4 C HTM $2.25 \mathrm{~Hz}$

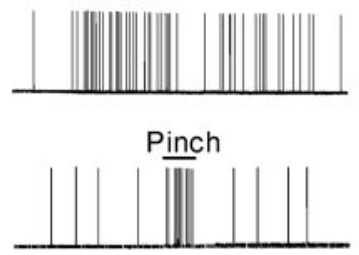

Figure 6. The rate of spontaneous firing in nociceptors in nerve injury and CFA rats. $\boldsymbol{A}$, Significantly higher median SA rate in L4 C-fiber nociceptive-type neurons in mSNA than SNA rats (Mann-Whitney test, two-tailed) and significantly higher median $S A$ rate $1 \mathrm{~d}$ compared with $4 \mathrm{~d}$ after CFA (Mann-Whitney test, one-tailed). $\boldsymbol{B}$ shows that, in L4 A-fiber nociceptors, there was no significant difference between median rate in $\mathrm{mSNA}$ rats $(n=7 ; 2 \mathrm{~A} \delta$ and $5 \mathrm{~A} \alpha \beta$ cells) compared with SNA rats ( $n=4 ; 1 \mathrm{~A} \delta$ and $3 \mathrm{~A} \alpha / \beta$ neurons). Ipsil means ipsilateral to nerve injury or CFA injection. $C$ and $D$ show example records of two typical $L 4 C$-fiber nociceptors firing spontaneously (top traces) at $0.02 \mathrm{~Hz}$ in SNA (C) and $2.25 \mathrm{~Hz}$ in mSNA (D) rats and, on bottom traces, show the same neurons responding to noxious pinch (note different timescale). Both neurons had subcutaneous (deep) receptive fields and did not respond to non-nociceptive stimuli including brush, touch, pressure, and tap (data not shown). ${ }^{*} p<0.05$; ${ }^{* *} p<0.01$.

throughout. These are 1.9 and $0.24 \mathrm{~Hz}$ for mSNA and SNA, respectively; comparing these values provides a more conservative estimate of nearly an eightfold greater number of spikes per second in mSNA rats. Figure $6, C$ and $D$, shows records from two typical L4 C-fiber nociceptors (HTMs) firing spontaneously at $0.02 \mathrm{~Hz}$ in SNA and $2.25 \mathrm{~Hz}$ in mSNA rats, and in bottom traces on a different timescale, the response of each unit to a noxious pinch is shown.

\section{A-fiber neurons}

In contrast, in L4 A-fiber nociceptors, the median firing rate was slightly, but not significantly, lower, in mSNA (median, $0.01 \mathrm{~Hz}$; mean, $0.035 \mathrm{~Hz}$ ) than in SNA rats (median, $0.035 \mathrm{~Hz}$; mean, 0.17 $\mathrm{Hz}$ ) (Fig. 6B).

\section{Comparison of SA rate in $\mathrm{L} 4 \mathrm{C}$ - and A-fiber nociceptors in mSNA rats}

From the above, in mSNA rats, the spontaneous firing rate of L4 $\mathrm{C}$-fiber nociceptors was higher than that of L4 A-fiber nociceptors by 180 -fold if medians are compared $(\mathrm{C}, 1.8 \mathrm{~Hz}$, vs A, 0.01 $\mathrm{Hz} ; p<0.01)$ or by $>50$-fold if means are compared $(\mathrm{C}, 1.9 \mathrm{~Hz}$, vs A, $0.035 \mathrm{~Hz}$ ) (Fig. 6A, B; Table 1). 
Table 1. Estimates of total spontaneous input to the CNS

\begin{tabular}{|c|c|c|c|c|c|c|c|}
\hline \multirow[b]{2}{*}{ Type of neuron } & \multicolumn{2}{|c|}{ Firing rate per neuron } & \multicolumn{2}{|c|}{ Calculated no. SD and LL neurons } & \multirow[b]{2}{*}{$\begin{array}{l}\% \text { NOCIS } \\
\text { with SA }\end{array}$} & \multicolumn{2}{|c|}{ Calculated total } \\
\hline & $\begin{array}{l}\text { Median } \\
(\mathrm{Hz})\end{array}$ & $\begin{array}{l}\text { Mean } \\
(\mathrm{Hz})\end{array}$ & Total & NOCIS & & $\begin{array}{l}\text { No. NOCIS } \\
\text { with SA }\end{array}$ & $\begin{array}{l}\text { Spinal cord } \\
\text { input }(\mathrm{Hz})\end{array}$ \\
\hline \multicolumn{8}{|l|}{ Nerve injury } \\
\hline C-NOCIS mSNA L4 & 1.8 & 1.9 & 8400 SD & 7560 & 35 & 2646 & 5027 \\
\hline C-NOCIS SNA L4 & 0.017 & 0.24 & 8400 SD & 7560 & 35 & 2646 & 635 \\
\hline A-NOCIS mSNA L4 & 0.01 & 0.035 & $3600 \mathrm{LL}$ & 828 & 10.3 & 85 & 3 \\
\hline A-NOCIS SNA L4 & 0.035 & 0.17 & $3600 \mathrm{LL}$ & 828 & 14.3 & 118 & 20 \\
\hline \multicolumn{8}{|c|}{ CFA-induced inflammation } \\
\hline CFA1 L4 + L5 & 0.5 & 0.59 & 21,000 SD & 18,900 & 38 & 7182 & 4237 \\
\hline \multirow[t]{2}{*}{ CFA4 L4 + L5 } & 0.1 & 0.15 & 21,000 SD & 18,900 & 25 & 4725 & 709 \\
\hline & & a & $\mathrm{b}$ & c & $d$ & $e=c \times d$ & $f=a \times e$ \\
\hline
\end{tabular}

For SNA and mSNA rats, the spontaneous firing rate was counted in individual L 4 nociceptive neurons with SA (see Results). For (FA rats, calculations are for L4 and L5, because both DRGs project to the inflamed hindlimb. The mean firing rate (a) (not the median) was used as the appropriate variable for estimating total firing rate. The derivation of the numbers is described in detail in Results. The calculated total numbers of neurons (columns b and c) are derived from published data. The "\% NOCIS with SA" (d) was measured in this study. The calculated totals of neuronal activity are derived as shown in e and f. N.B., Although these are estimates, we have kept exact numerical values to make calculations easier to follow. SD, Small dark neurons that have C-fibers; LL, large light neurons that have A-fibers (Harper and Lawson, 1985); NOCIS, nociceptive-type neurons.

\section{SA in CFA-treated rats}

The median firing rates of C-fiber nociceptors with SA were significantly greater at $1 \mathrm{~d}$ than at $4 \mathrm{~d}$ post-CFA (Fig. $6 \mathrm{~A}$ ), consistent with the possibility that a certain firing rate in these neurons, or total level of input along the SN, might be required for SFL to occur. An ongoing/spontaneous firing rate of $0.59 \mathrm{~Hz}$ (Fig. 6, Table 1) in approximately one-third of C-fiber nociceptors in L4 and L5 DRGs $1 \mathrm{~d}$ after CFA appears to be enough to cause a small amount of SFL, whereas $0.15 \mathrm{~Hz} 4 \mathrm{~d}$ after CFA was not. Thus, a common feature between the rats with SFL in CFA1 and mSNA models is a high rate of spontaneous firing in C-fiber nociceptors.

\section{Estimate of total CNS input from L4 C- and A-fiber SA \\ SNA and $m S N A$ rats}

Assuming the above values for incidence and rate of SA are representative of the whole L4 DRG, we have estimated the likely magnitudes of total C- and A-fiber spontaneous nociceptive input into the spinal cord along the L4 dorsal root after these nerve injuries as follows. In rat L5 DRGs, there are 18,000 neurons, as estimated using the dissector method (Tandrup, 1993). The rat L4 DRG is smaller than L5 with $\sim 68 \%$ of the number of L5 neurons (Arvidsson et al., 1986); together, these values indicate that L4 should have $\sim 12,000$ neurons. Small dark (SD) DRG neurons mostly/all have C-fibers, and large light (LL) neurons have A-fibers (Harper and Lawson, 1985). Counts with the dissector method indicate that, in rat lumbar DRGs, the ratio of small dark to large light DRG neurons is 7:3 (Tandrup, 1993). This ratio indicates that, in L4 DRGs, there should be $\sim 8400$ small dark neurons with C-fibers and 3600 large light neurons with A-fibers (Table 1, b).

Our recent studies (Fang et al., 2005b) indicate that, for cutaneous and subcutaneous limb afferents in L4 and L5 DRGs, most $(>90 \%)$ afferent C-fiber neurons are nociceptors, whereas only $\sim 23 \%$ of afferent A-fiber neurons are nociceptors. If we assume that these values are correct for the whole L4 DRG, this gives a value of $\sim 7560 \mathrm{C}$-fiber nociceptive and $828 \mathrm{~A}$-fiber nociceptive neurons (Table 1, c). These values plus the percentages of $\mathrm{C}$ - and A-fiber nociceptors with SA (Table 1, d) enable estimation of the total numbers of nociceptors with SA in the L4 DRG (e). Finally, multiplying these numbers by the mean SA firing rate provides the estimated total rate of firing for all C-and all A-fiber nociceptors in L4 DRGs in SNA and mSNA rats (Table 1, f). Note that the values in Table 1, a and e, for A-fibers were not significantly different in SNA and mSNA rats, and thus the final values of 20 and 3 in column $\mathrm{f}$ do not represent significantly different levels of input. The total firing rates (f) highlight the much greater C-fiber input in mSNA rats than in SNA rats (eightfold greater). The lack of increased SA in A-fiber nociceptors in mSNA than in SNA rats plus the much greater spontaneous firing rate in L4 C-fiber compared with A-fiber nociceptors in mSNA rats indicate that A-fiber nociceptor firing in the L4 DRG is unlikely to contribute significantly to the generation of SFL in mSNA rats.

\section{CFA-treated rats}

Using the same basis for estimation of the afferent barrage as for the neuropathic pain models as above, the C-fiber input along the SNs is calculated in Table 1. For this purpose, the total number of neurons in L5 is taken to be 18,000 and the total for L4 and L5 together to be 30,000 (see above). At $1 \mathrm{~d}$ after CFA, the total input to the spinal cord in L4 and L5 spinal nerves would be $4.2 \mathrm{kHz}$, some six times greater than the input at $4 \mathrm{~d}$ after administration of CFA (Table 1).

Because of the high proportion of intact $\mathrm{A} \delta$-nociceptors with SA (6 of 10) (Fig. 5) $1 \mathrm{~d}$ after CFA, we considered the total CNS input from these neurons. The firing rates of these $6 \mathrm{~A} \delta$ units with SA (range, 0.03-0.6 Hz; mean, 0.23; median, $0.18 \mathrm{~Hz}$ ) were lower than those in the C-fiber units with SA. We estimated (as above) the $A \delta$-fiber nociceptor afferent barrage in CFA1 rats in ipsilateral L4 plus L5 DRGs. The number of large light neurons is $\sim 9000(30,000 \times 0.3)$ (see above). If $23 \%$ of these are nociceptive (see above), and the proportion of A-fiber nociceptors with $\mathrm{A} \delta$ fibers is $34 \%$ in rat (Djouhri and Lawson, 2004), then there should be $\sim 700$ A $\delta$ nociceptors in these two DRGs together. Of these, $60 \%$ show SA (that is, $\sim 420$ neurons). A mean firing rate of $0.23 \mathrm{~Hz}$ in 420 neurons results in a total firing rate of $\sim 100 \mathrm{~Hz}$ $(96.6 \mathrm{~Hz})$. Although this low value does not exclude an important role SFL for specifically targeted A $\delta$ inputs, it does represent a 40 -fold smaller barrage of information $(<0.1 \mathrm{kHz})$ compared with the C-fiber input $(>4 \mathrm{kHz}$ ) (Table 1$)$. We did not record the proportion of $A \alpha / \beta$ fiber nociceptors with SA in CFA1 rats.

Calculating the total CNS input is an approximate estimate, with errors (the levels of which can be found in the cited papers) at all stage of the calculations. Nonetheless, because the errors are similar for all models, it is a valid comparison of approximate total inputs in the different models.

\section{Relationships between SFL and rate of spontaneous firing in C-nociceptors}

If faster firing in intact C-nociceptors is necessary for SFL, then the extent of SFL may be related to the firing rate. First, we compared these in individual rats. We had SFL records for only 13 rats in which successful C-fiber recordings were made. (CFA4 data 


\section{SFL and C-neurons SA rate}

\section{A Individual rats}

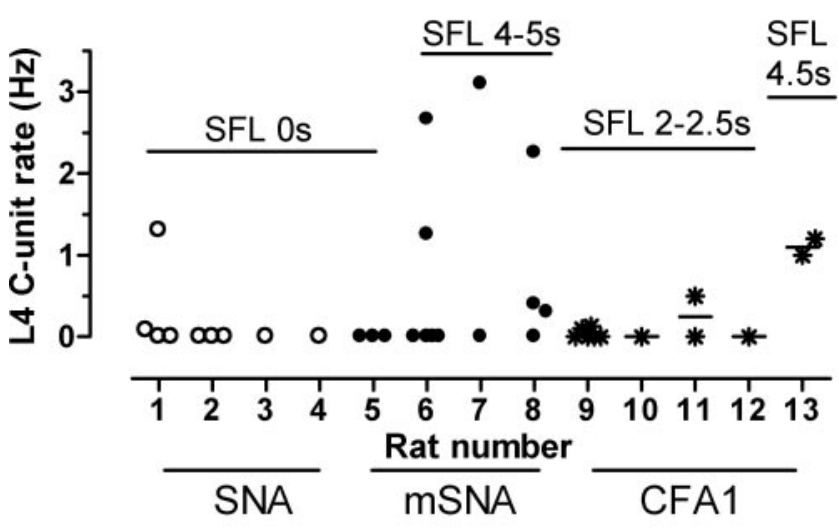

\section{B Individual rats}

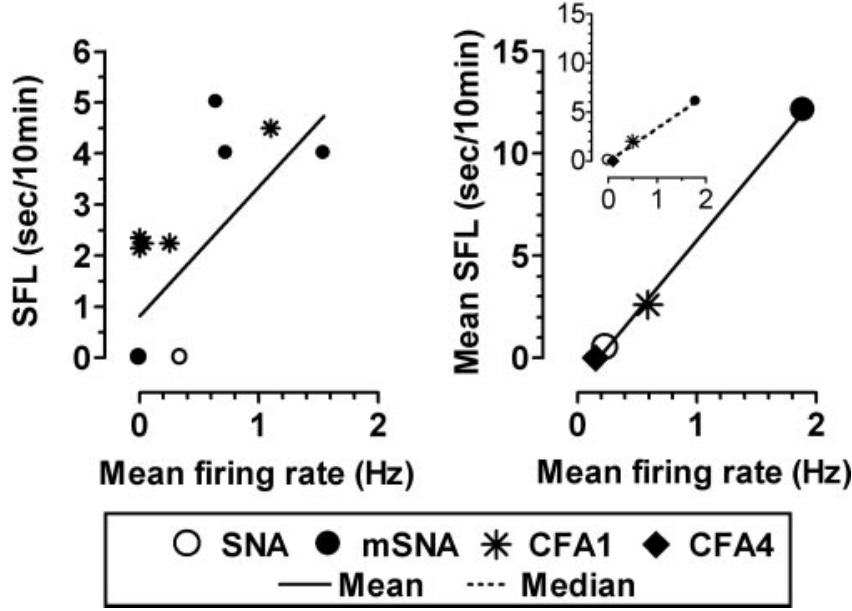

Figure 7. Relationships between SFL and SA rate in C-nociceptors. $A, A$ scattergraph of individual $C$-fiber firing rate recorded in different rats ( 1 rat per column; the number beneath is the rat number), grouped by treatment [SNA, open circles; mSNA, filled circles; (FA (1 d), asterisks]. SFL duration is indicated above the columns. Each symbol is one C-fiber neuron. $\boldsymbol{B}$, The SFL for each rat in $\boldsymbol{A}$ is plotted against the mean firing rate for $C$-nociceptor-type neurons (including units with and without $\mathrm{SA}$ ) in that rat. There is a highly significant linear correlation $\left(p<0.01 ; r^{2}=0.468\right)$ between rate and SFL. C, Plot of mean SFL duration in seconds per 10 min for SNA, mSNA, CFA1, and CFA4 rats (from data plotted in Fig. $6 A$ ) against mean firing rates (from data plotted in Fig. $2 A$ ) of (-fiber nociceptor type neurons with SA for that group. There is a highly significant correlation whether the means (main graph; $p<0.001, r^{2}=0.998$ ) or medians (inset graph; $p<005, r^{2}=0.991$ ) are correlated. The symbols below relate to all graphs; the lines indicate significant correlation.

are not included, because SFL was not measured in the same rats. N.B., No foot lifting was ever seen in CFA4 rats, and SA firing rates can be seen in Fig. 6.) In Figure 7A, we compare the SFL and SA firing rates for each of these 13 rats. Note that the firing rate was low or 0 in rats with no SFL even for an mSNA rat (rat 5) and higher firing rates were seen in rats with SFL, in both mSNA and in CFA1 rats. The mean firing rate for each rat was plotted against the SFL duration (Fig. 7B). A highly significant linear correlation indicates that, for these 13 rats, the SFL was related to the mean $\mathrm{C}$-neuron firing rate (including neurons with and without SA).

We also determined, for each of the four groups, CFA1, CFA4, SNA, and mSNA, the mean and median amount of SFL (in sec- onds per $10 \mathrm{~min}$ ) and the mean rate of spontaneous firing in C-fiber neurons with SA for that group, providing us with one point summarizing the values for that whole group. For this, we used all the SFL data in Figure 2 and the values for firing rate given in Figure 6 and Table 1. Interestingly, linear regression analysis shows that SFL is highly significantly related to both the mean and median L4 C-fiber firing rate (Fig. 7C). Extrapolation from small numbers of DRG neurons should be interpreted with caution. However, we believe these neurons are randomly sampled and thus representative of the total population of neurons within the DRGs examined. The patterns that emerge from all the evidence together are persuasive and can be used to generate hypotheses for future testing.

\section{Discussion}

This is the first report of a link between the amount of spontaneous firing in intact C-fiber nociceptors and spontaneous pain (indicated by SFL) in neuropathic and inflammatory pain models. This link is based on the following: (1) significant SFL in mSNA but not SNA rats, (2) the lack of correlation between evoked pain behaviors and SFL in mSNA rats, suggesting spontaneous pain rather than allodynia as a probable cause of SFL, (3) greater spontaneous firing rates in $\mathrm{L} 4 \mathrm{C}$-nociceptors in $\mathrm{mSNA}$ than SNA rats, but (4) similar percentages of L4 C-nociceptors with SA in mSNA and SNA rats. Studies after CFA-induced inflammation, confirm that (1) inflammation induces SA in C-nociceptors and (2) SFL at $1 \mathrm{~d}$ is associated with faster C-fiber SA induced by inflammation. The correlation between C-fiber SA firing rate and SFL duration in both inflammatory and neuropathic models suggests this firing rate is an important drive for SFL.

\section{SFL as a behavioral sign of spontaneous pain}

The lack of correlation between SFL and evoked pain behaviors (allodynia or hyperalgesia) in mSNA rats suggests that SFL in these rats results not from allodynia attributable to contact but from spontaneous pain.

It is not known how much SFL (how many seconds per 10 $\min$ ) is required to indicate spontaneous pain. An afferent input strong enough to override normal locomotory/exploratory behavior would be needed for an animal to lift/examine its foot; even a few seconds of SFL probably indicates substantial spontaneous pain as would be the case in humans. In mSNA rats, the consistent presence of SFL, the relatively short SFL duration, and generally normal behavior indicate this to be a model reliably causing spontaneous pain behavior but with only mild-moderate discomfort. However, distractions should be limited during SFL measurement, because they probably cause greater variability in responses to ongoing rather than evoked, synchronized, afferent input.

Our findings of increased percentage of L4 C-fiber neurons with SA 1 week after L5 SN injury agrees with previous studies in rat (Wu et al., 2001) and monkey (Ali et al., 1999). The lower values in our study (35\%) than that of Wu et al. (>50\%) may relate to strain differences and/or shorter initial SA observation periods in our study (2 vs $5 \mathrm{~min}$ ) that could exclude units with very low firing rates ( $<1$ in $2 \mathrm{~min}$ ).

Two additional studies (Boucher et al. 2000; Ma et al. 2003) failed to demonstrate SA in spared L4 C-fibers after L5 SN injury, perhaps because (1) Boucher et al. (2000) transected the L4 SN just before dorsal root fiber recordings, thus preventing SA originating in C-fibers distal to the transection reaching the dorsal roots; (2) Ma et al. (2003) used postoperative antibiotics. These 
would reduce inflammatory responses to bacterial infection (if present despite aseptic operating conditions), or may directly influence inflammatory responses (Hamilton-Miller, 2001) such as that to chromic gut.

The similar proportion (one-third) of L4 C nociceptive-type neurons showing SA in both SNA and mSNA rats suggests involvement of the same C-fiber neuron population in both models. However, the large increase in frequency of spontaneous firing in L4 C-fiber nociceptive-type neurons in mSNA compared with SNA rats suggests that effects of chromic gut add to those of L5 axotomy, resulting in increased firing rates in this same group of neurons.

This study does not address the role of central sensitization. The higher rate of firing $(\sim 2 \mathrm{~Hz})$ in L4 C-fiber neurons in mSNA rats, which translates into $5 \mathrm{kHz}$ along the $\mathrm{L} 4$ dorsal root, may well be enough to trigger SFL in the absence of central sensitization. However, this barrage may also cause central sensitization (Woolf and Wall, 1986), which could enhance its effectiveness in generating SFL/spontaneous pain. It seems unlikely that A-fiber nociceptors make a significant contribution to SFL in mSNA rats, because the frequency of firing is very low and the proportion with SA remains low. However, we cannot exclude an effect of even a small A-fiber input, especially if enhanced by central sensitization.

\section{Inflammation as a cause of SA in intact L4 C-fibers}

There are similarities in the L4 intact C-nociceptors after nerve injury and CFA treatment that suggest a common inflammatory mechanism in both cases. These include the following: (1) the proportion with SA and (2) an increase in action potential height (Djouhri, Fang, McMullan, and Lawson, unpublished observations). The positive correlation between SA firing rate in C-nociceptors and SFL, in both inflammatory and nerve injury models, is strongly suggestive of a link between these variables in both types of model.

Our findings lend support to the growing evidence for inflammatory mediators influencing sensory neurons and having a role in generation of neuropathic pain (for review, see Li et al., 2000; Watkins and Maier, 2002; Lawson, 2005). Wallerian degeneration of peripheral nerve fibers causes an inflammatory response (Shamash et al., 2002), which is thought to be the cause of SA in intact C-fiber afferents running in the same peripheral nerve ( $\mathrm{Li}$ et al. 2000; Wu et al. 2001). Evidence of a role for inflammatory mediators includes the following. After CCI, SA in DRG neurons is markedly increased by inflammatory mediators (Song et al., 2003). In another neuropathic pain model, TNF $\alpha$ (tumor necrosis factor $\alpha$ ), a proinflammatory cytokine, causes increased dorsal root SA in C-fibers and increased SFL when applied to the DRG (Schafers et al., 2003). Nerve growth factor (NGF) plays a key role in the response of sensory neurons to inflammation; inflamed peripheral tissues upregulate NGF (Woolf et al. 1994), which leads to NGF-dependent SA in C-fiber DRG neurons (Djouhri et al. 2001), probably because NGF influences neuronal expression of ion channels or receptors that contribute to SA generation (Lawson, 2005). NGF levels are raised in adjacent spared L4 DRG neuronal somata after L5 axotomy, possibly because of raised NGF in peripheral nerve during Wallerian degeneration (Shamash et al., 2002) and reduced competition for target-derived NGF by axotomized L 5 fibers allowing increased NGF uptake by L4 fibers (Obata et al., 2004). Nociceptive DRG neurons express high levels of trkA (the high affinity NGF receptor) (Fang et al. 2005a) and can therefore take up and be influenced by NGF.

Possible causes of inflammatory influences on intact L4 neu- rons in SNA and mSNA nerve injury models are summarized in Figure 1. Inflammation 1 and increased NGF availability in skin are thought to result from degenerating L5 fibers (see above) and inflammation 2 is chromic gut induced (see Introduction). Inflammation 1 alone (SNA) results in a lower rate of SA than inflammations 1 plus 2 (mSNA). Alone neither inflammation 1 (SNA) nor inflammation 2 (after L4 chromic-gut loose ligation only) (S. Koutsikou and S. N. Lawson, unpublished observations) causes significantly increased SFL. Cumulative effects of inflammation 1 (perhaps with increased NGF availability in the periphery) plus inflammation 2 thus appear to be required for both the SFL and the faster SA rate in intact L4 C-fibers in mSNA rats.

\section{Sensations that may result from C-fiber SA}

The different types of spontaneous pain sensation probably depend on the central projections of spontaneously active afferent fibers. Because activation of $\mathrm{C}$ - and A-fiber nociceptors generate unpleasant/unbearable burning and sharp pain, respectively (for review, see Djouhri and Lawson, 2004), SA in these two fiber groups may evoke, respectively, the burning and stabbing spontaneous pain reported in human patients. Thus, SA in intact C-fiber neurons reported here may cause burning spontaneous pain. It is not known whether A-fiber nociceptors are responsible for stabbing spontaneous pain, and, if so, whether these have axotomized or intact fibers. However, because SA in $\mathrm{A} \delta$ nociceptors was induced by peripheral inflammation but not nerve injury or inflammation applied to the nerve (chromic gut), the myelin sheath may normally protect such fibers from inflammatory mediators except in the periphery or possibly after demyelination.

In summary, we present evidence linking high spontaneous firing rates in intact C-fiber nociceptive neurons with SFL in both neuropathic and inflammatory pain models. In neuropathic models, a low spontaneous firing rate in these neurons, possibly resulting from inflammatory effects of degenerating axotomized L5 fibers, is not associated with significant SFL. Significant SFL is accompanied by the higher firing rate that appears to depend on added inflammatory influences provided in this mSNA model by the chromic gut L4 SN ligation. This plus the overall correlations between SFL and C-nociceptor firing rate after nerve injury and CFA treatment lead us to propose that spontaneous neuropathic pain may result from cumulative neuroinflammatory influences not necessarily all at the same site on the neuron, causing relatively rapid spontaneous firing of intact $\mathrm{C}$-nociceptors. Our findings therefore support the view, based on the high percentage (50\%) of clinical neuropathic pain cases associated with infection/inflammation of peripheral nerves (Said and HontebeyrieJoskowicz, 1992), that inflammation is important in neuropathic pain (Devor and Seltzer, 1999; Watkins and Maier, 2002). Importantly, we also suggest an underlying neuronal mechanism linking inflammation, rate of spontaneous firing, and spontaneous pain. Elimination of SA in these intact C-fiber nociceptors may therefore relieve spontaneous neuropathic pain. Additional studies are needed to elucidate the altered electrophysiological properties and/or chemical influences causing this C-fiber SA.

\section{References}

Ali Z, Ringkamp M, Hartke TV, Chien HF, Flavahan NA, Campbell JN, Meyer RA (1999) Uninjured C-fiber nociceptors develop spontaneous activity and alpha-adrenergic sensitivity following L6 spinal nerve ligation in monkey. J Neurophysiol 81:455-466.

Amir R, Michaelis M, Devor M (1999) Membrane potential oscillations in dorsal root ganglion neurons: role in normal electrogenesis and neuropathic pain. J Neurosci 19:8589-8596. 
Arvidsson J, Ygge J, Grant G (1986) Cell loss in lumbar dorsal root ganglia and transganglionic degeneration after sciatic nerve resection in the rat. Brain Res 373:15-21.

Attal N, Bouhassira D (2004) Neuropathic pain: experimental advances and clinical applications. Rev Neurol (Paris) 160:199-203.

Bennett GJ, Xie YK (1988) A peripheral mononeuropathy in rat that produces disorders of pain sensation like those seen in man. Pain 33:87-107.

Bonica JJ (1990) Evolution and current status of pain programs. J Pain Symptom Manage 5:368-374.

Boucher TJ, Okuse K, Bennett DL, Munson JB, Wood JN, McMahon SB (2000) Potent analgesic effects of GDNF in neuropathic pain states. Science 290:124-127.

Chaplan SR, Bach FW, Pogrel JW, Chung JM, Yaksh TL (1994) Quantitative assessment of tactile allodynia in the rat paw. J Neurosci Methods 53:55-63.

Choi Y, Yoon YW, Na HS, Kim SH, Chung JM (1994) Behavioral signs of ongoing pain and cold allodynia in a rat model of neuropathic pain. Pain 59:369-376.

Devor M, Seltzer Z (1999) Pathophysiology of damaged nerves in relation to chronic pain. In: Textbook of pain (Wall PD, Melzack R, eds), pp 129163. London: Churchill Livingstone.

Djouhri L, Lawson SN (2001) Increased conduction velocity of nociceptive primary afferent neurons during unilateral hindlimb inflammation in the anaesthetised guinea-pig. Neuroscience 102:669-679.

Djouhri L, Lawson SN (2004) Abeta-fiber nociceptive primary afferent neurons: a review of incidence and properties in relation to other afferent A-fiber neurons in mammals. Brain Res Brain Res Rev 46:131-145.

Djouhri L, Bleazard L, Lawson SN (1998) Association of somatic action potential shape with sensory receptive properties in guinea-pig dorsal root ganglion neurones. J Physiol (Lond) 513:857-872.

Djouhri L, Dawbarn D, Robertson A, Newton R, Lawson SN (2001) Time course and nerve growth factor dependence of inflammation-induced alterations in electrophysiological membrane properties in nociceptive primary afferent neurons. J Neurosci 21:8722-8733.

Fang X, Djouhri L, Black JA, Dib-Hajj SD, Waxman SG, Lawson SN (2002) The presence and role of the tetrodotoxin-resistant sodium channel $\mathrm{Na}(\mathrm{v}) 1.9(\mathrm{NaN})$ in nociceptive primary afferent neurons. J Neurosci 22:7425-7433.

Fang X, Djouhri L, McMullan S, Berry C, Okuse K, Waxman SG, Lawson SN (2005a) trkA is expressed in nociceptive neurons and influences electrophysiological properties via Nav1.8 expression in rapidly conducting nociceptors. J Neurosci 25:4868-4878.

Fang X, McMullan S, Lawson S, Djouhri L (2005b) Electrophysiological differences between nociceptive and non-nociceptive somatic DRG neurones in the rat in vivo. J Physiol (Lond) 565:927-943.

Hamilton-Miller JM (2001) Immunopharmacology of antibiotics: direct and indirect immunomodulation of defence mechanisms. J Chemother 13:107-111.

Han HC, Lee DH, Chung JM (2000) Characteristics of ectopic discharges in a rat neuropathic pain model. Pain 84:253-261.

Hargreaves K, Dubner R, Brown F, Flores C, Joris J (1988) A new and sensitive method for measuring thermal nociception in cutaneous hyperalgesia. Pain 32:77-88.

Harper AA, Lawson SN (1985) Conduction velocity is related to morphological cell type in rat dorsal root ganglion neurones. J Physiol (Lond) 359:31-46.

Kim KJ, Yoon YW, Chung JM (1997) Comparison of three rodent neuropathic pain models. Exp Brain Res 113:200-206.

Kim SH, Chung JM (1992) An experimental model for peripheral neuropathy produced by segmental spinal nerve ligation in the rat. Pain 50:355-363.

Koutsikou S, Lawson SN (2002) Increased reliabilty in a modified spinal nerve injury rat model of neuropathic pain, involving L5 transection plus L4 loose ligation. J Physiol (Lond) 543:113P.

Lawson SN (2005) The peripheral sensory nervous system: dorsal root gan- glion neurons. In: Peripheral neuropathy (Dyck PJ, Thomas PK, eds), pp 163-202. Philadelphia: Saunders.

Lee DH, Iyengar S, Lodge D (2003) The role of uninjured nerve in spinal nerve ligated rats points to an improved animal model of neuropathic pain. Eur J Pain 7:473-479.

Li Y, Dorsi MJ, Meyer RA, Belzberg AJ (2000) Mechanical hyperalgesia after an $\mathrm{L} 5$ spinal nerve lesion in the rat is not dependent on input from injured nerve fibers. Pain 85:493-502.

Liu CN, Wall PD, Ben Dor E, Michaelis M, Amir R, Devor M (2000) Tactile allodynia in the absence of $\mathrm{C}$-fiber activation: altered firing properties of DRG neurons following spinal nerve injury. Pain 85:503-521.

Ma C, Shu Y, Zheng Z, Chen Y, Yao H, Greenquist KW, White FA, LaMotte RH (2003) Similar electrophysiological changes in axotomized and neighboring intact dorsal root ganglion neurons. J Neurophysiol 89:1588-1602.

Mao J, Price DD, Mayer DJ, Lu J, Hayes RL (1992) Intrathecal MK-801 and local nerve anesthesia synergistically reduce nociceptive behaviors in rats with experimental peripheral mononeuropathy. Brain Res 576:254-262.

Mashima T, Udagawa S, Tsuruo T (2001) Involvement of transcriptional repressor ATF3 in acceleration of caspase protease activation during DNA damaging agent-induced apoptosis. J Cell Physiol 188:352-358.

Maves TJ, Pechman PS, Gebhart GF, Meller ST (1993) Possible chemical contribution from chromic gut sutures produces disorders of pain sensation like those seen in man. Pain 54:57-69.

Michaelis M, Liu X, Janig W (2000) Axotomized and intact muscle afferents but no skin afferents develop ongoing discharges of dorsal root ganglion origin after peripheral nerve lesion. J Neurosci 20:2742-2748.

Obata K, Yamanaka H, Dai Y, Mizushima T, Fukuoka T, Tokunaga A, Yoshikawa H, Noguchi K (2004) Contribution of degeneration of motor and sensory fibers to pain behavior and the changes in neurotrophic factors in rat dorsal root ganglion. Exp Neurol 188:149-160.

Said G, Hontebeyrie-Joskowicz M (1992) Nerve lesions induced by macrophage activation. Res Immunol 143:589-599.

Schafers M, Lee DH, Brors D, Yaksh TL, Sorkin LS (2003) Increased sensitivity of injured and adjacent uninjured rat primary sensory neurons to exogenous tumor necrosis factor- $\alpha$ after spinal nerve ligation. J Neurosci 23:3028-3038.

Shamash S, Reichert F, Rotshenker S (2002) The cytokine network of Wallerian degeneration: tumor necrosis factor- $\alpha$, interleukin- $1 \alpha$, and interleukin-1 $\beta$. J Neurosci 22:3052-3060.

Song XJ, Zhang JM, Hu SJ, LaMotte RH (2003) Somata of nerve-injured sensory neurons exhibit enhanced responses to inflammatory mediators. Pain 104:701-709.

Tandrup T (1993) A method for unbiased and efficient estimation of number and mean volume of specified neuron subtypes in rat dorsal root ganglion. J Comp Neurol 329:269-276.

Tsujino H, Kondo E, Fukuoka T, Dai Y, Tokunaga A, Miki K, Yonenobu K, Ochi T, Noguchi K (2000) Activating transcription factor 3 (ATF3) induction by axotomy in sensory and motoneurons: a novel neuronal marker of nerve injury. Mol Cell Neurosci 15:170-182.

Watkins LR, Maier SF (2002) Beyond neurons: evidence that immune and glial cells contribute to pathological pain states. Physiol Rev 82:981-1011.

Woolf CJ, Wall PD (1986) Relative effectiveness of C primary afferent fibers of different origins in evoking a prolonged facilitation of the flexor reflex in the rat. J Neurosci 6:1433-1442.

Woolf CJ, Safieh-Garabedian B, Ma QP, Crilly P, Winters J (1994) Nerve growth factor contributes to the generation of inflammatory sensory hypersensitivity. Neuroscience 62:327-331.

Wu G, Ringkamp M, Hartke TV, Murinson BB, Campbell JN, Griffin JW, Meyer RA (2001) Early onset of spontaneous activity in uninjured C-fiber nociceptors after injury to neighboring nerve fibers. J Neurosci 21:RC140(1-5).

Yoon YW, Na HS, Chung JM (1996) Contributions of injured and intact afferents to neuropathic pain in an experimental rat model. Pain 64: $27-36$. 